\title{
Can hospital use be a measure of need for health care?
}

\author{
MYFANWY MORGAN, NICHOLAS MAYS, AND WALTER W HOLLAND \\ From the Department of Community Medicine, United Medical and Dental Schools, St Thomas's Hospital, London \\ SE1
}

To achieve an equitable distribution of health service resources between geographical areas requires a measure of the relative needs of populations. For 11 years the methods of the Resource Allocation Working Party (RAWP) have been employed in England for this purpose. ${ }^{1}$ RAWP uses mortality rates in the form of standardised mortality ratios (SMRs) as a proxy for morbidity, to take account of differences in the relative needs of populations for hospital care in excess of those 'explained' by the age/sex structure of the population. In this way RAWP aims gradually to redistribute National Health Service (NHS) resources more fairly in relation to need at both Regional and sub-Regional levels.

A major criticism of the RAWP formula has centred on its use of SMRs as an indicator of need for hospital care. ${ }^{2-4}$ In particular, it is argued that the relatively high level of morbidity experienced by 'deprived' populations may not be adequately reflected in their SMRs. Hospitals in deprived areas may also experience greater demands on their services at similar levels of morbidity, arising from the populations' social needs for in-patient care due to their poor housing conditions, living alone, etc. The greater needs of deprived populations have their major impact at a sub-Regional level. This is due to the concentration of deprived areas in some inner city health Districts.

The Thames Regions, which include a number of deprived inner city Districts, have responded to the perceived inadequacies of SMRs in subregional RAWP by using socioeconomic classifications as measures of need for hospital care. Their approach has been to examine rates of hospital use by different socioeconomic groups to generate 'deprivation weights' as opposed to mortality weights. Recently, the NHS Management Board Review of National RAWP has called for research along similar lines in the hope of improving the needs element in the RAWP formula. ${ }^{5}$

Current interest in developing measures of need based on rates of hospital use raises questions concerning the interpretation of utilisation data and its relation to population need. Of particular significance are the large geographical variations in utilisation rates. For example, McPherson and colleagues found that rates of hospital utilisation for common surgical procedures (standardised by age and sex) varied as much as twofold within England and Wales and up to sevenfold between England and Wales, Canada, and the United States. ${ }^{6}$ Data assembled by the London Health Planning Consortium (LHPC) similarly show marked variations in hospital admission rates for all acute specialties (excluding Regional acute specialties) between the 14 Regions in England. For example, there was a $25 \%$ difference in admission rates between the Trent and Yorkshire Regions. Although the four Thames Regions all had relatively high admission rates, there was considerable variation within these Regions, with 115 admissions per 1000 resident population in inner London, 100 per 1000 in outer London, and 86 per 1000 in the remainder of the four Thames Regions. This compares with a figure of 91 per 1000 in England as a whole. An analysis of admissions to medical specialties in the 15 health districts in South East Thames Region similarly shows that rates range from 19 to 57 admissions per 1000 population aged 0-14 years, from 41 to 81 per 1000 population aged 65-74 years, and from 35 to 119 per 1000 population aged 75 and over. ${ }^{8}$ In addition to these geographical variations in admission rates are important variations in the length of time patients spend in hospital in different areas of the country.9-10

Differences in rates of hospital use between Regions and Districts are partly accounted for by differences in morbidity, associated with the economic circumstances and social conditions of their populations. However, variations in overall hospitalisation rates appear too large to be accounted for by differences in morbidity alone. Studies of operation rates for particular conditions, such as hysterectomy, ${ }^{11}$ glue ear ${ }^{12}$ and cataract extraction, ${ }^{13}$ also indicate that the substantial geographical variations cannot be explained entirely by differences in the prevalence of these disorders.

\section{Variations in supply}

A key factor identified as contributing to the 
geographical variations in hospital use both between and within countries is the influence of health service provision, in terms of the supply of hospital beds and medical manpower. ${ }^{6,12}$ At a Regional level, the LHPC $^{7}$ identified the supply of beds as the major determinant of variations in hospital admission rates for acute (non-Regional) specialties. The LHPC also noted that, not surprisingly, the same relation occurs within Regions, with a correlation coefficient of +0.85 between hospital admission rates and available beds per 1000 resident population in health Districts within the four Thames Regions. There is also a general relation between length of stay and level of bed provision, the Region with consistently long lengths of stay (Mersey) having the most beds per 1000 population and the Region with consistently shorter stays (Oxford) having the fewest beds per 1000 population. ${ }^{5}$

Population needs for hospital services and the supply of beds are largely reconciled in the NHS by the medical profession, which, as Cooper ${ }^{15}$ notes, "appears to reassess its conception of need in line with current levels of provision". In the UK, these adjustments frequently originate in the referral decisions taken by general practitioners (GPs). In situations where hospital beds are in relatively plentiful supply and the population has easy access to these facilities (in terms of distance, time, and travel cost), general practitioners appear more likely to refer patients to outpatient departments for conditions that would otherwise be managed on a primary care basis. For example, Acheson ${ }^{16}$ quotes a Regional range for new outpatient attendances of between 154 and 225 per 1000 population. Regions characterised by high outpatient referral rates tend to have high inpatient admission rates and, in turn, to be those with the highest levels of resources. Once the patient is referred to hospital, bed availability again influences the selection of cases requiring admission and the timing of discharge, as demonstrated in the early studies by Feldstein $^{17}$ and Logan et al. ${ }^{18}$ More recently, Wennberg, Freeman, and Culp ${ }^{19}$ have compared hospital use in Boston and New Haven, which are both served mainly by teaching hospitals and have similar demographic profiles, although the per capita bed provision is $52 \%$ higher in Boston. Hospital admission rates in Boston and New Haven showed little difference for conditions where the form of treatment is well defined, such as acute myocardial infarction, gastrointestinal haemorrhage, inguinal hernia repair, and prostatectomy. For these conditions, differences in hospital use were explained largely by variations in lengths of stay. The variation in admission rates occurred mainly for minor medical conditions in which the decision to admit can be discretionary (eg, medical back problems, adult simple pneumonia, adult diabetes, bronchitis and asthma) and for some minor surgical procedures which are frequently handled in an outpatient setting (eg, minor operations on knee, dental extractions, paediatric hernia repair). Other evidence of the influence of resource provision on practice is the significant association between the level of diagnostic radiological examinations and the relative level of funding between Regions in England. ${ }^{20}$

The socioeconomic composition of the population varies between areas with different levels of provision. For example, deprived populations in the UK tend to be concentrated in inner city Districts that have a relatively plentiful supply of hospital beds. The high rates of hospital use in these areas are thus a product of the population's high level of need and the high level of supply. In contrast, rural populations have lower levels of hospital provision and exhibit relatively low rates of hospital use. ${ }^{8}$

\section{Variations in professional and patient behaviours}

Supply factors, although having a major influence on professional definitions of needs for hospital care, do not fully account for variations in professionaf practices. For example, up to 25 -fold variation in GPO referral rates has been observed within fairly small areas, and even close colleagues in the same practice may behave in very different ways when confrontedo with treating the same patient population. ${ }^{21-23}$ These variations have been examined in relation to, for example, the GP's age, experience, postgraduate? training, list size, practice organisation, and diagnostic back-up, but have not been satisfactorily explained. ${ }^{24-25}$ In one of the few studies of GPs' decisions to refer to outpatient departments, Dowie ${ }^{26}$ identified three sets of factors which appeared to explain variations in referral rates: professional attributes, such as medical knowledge and judgement; knowledge of the health care system; and personal style and interaction with patients.

Variations in professional practice also occur at the hospital level. For example, Bloor and Venters ${ }^{27}$ in a study of small area variations in tonsillectomy and adenoidectomy rates in Scotland, identified the practice style of specialists as a key influence on the number of operations performed. The authors identified the existence of two groups of specialists: a low acceptor, low operator group, and a high acceptor, higher operator group. As Wennberg ${ }^{28}$ notes, there is considerable scope for variations in practice style, as for more than $80 \%$ of medical conditions and for large numbers of surgical conditions the need for hospitalisation is not clearly defined and there is little professional consensus as to the appropriate form of management. Since variation 
in style is an integral feature of medical practice, it is unlikely to form a major factor accounting for overall differences in hospitalisation rates between Regions. However, practice style can have a substantial influence on admission rates for particular conditions and procedures within small areas.

Patient behaviours are assumed to play a fairly limited role in contributing to differences in hospitalisation rates in the UK, opportunities for self referral being restricted mainly to attendance at the accident and emergency department. However, there is some evidence that women in non-manual classes present more frequently to the general practitioner for minor conditions than those in the working classes. ${ }^{29}$ Middle class patients also appear more likely to be referred for specialist advice, and the GP to order more laboratory tests for social classes I and II than for IV and $\mathrm{V}$ after controlling for differences in patient's age, diagnosis, and doctor. ${ }^{29.30}$ This raises questions of the appropriateness of different levels of investigation and the difference in overall demands made on the hospital service as a result of these variations in patient and physician behaviours.

\section{Alternative provision}

A further influence on the utilisation of NHS hospital beds is the availability of alternative facilities and services, and especially of alternative forms of inpatient care, such as convalescent hospitals and nursing homes. These facilities are not included in the Hospital Activity Analysis (HAA) produced by each Region. Employing HAA data to derive utilisation figures therefore means that no account is taken of needs catered for by these facilities, which may serve to reduce 'official' hospital admission rates or lengths of stay.

The provision of these alternative forms of care varies between Regions and Districts, private nursing home provision being particularly concentrated in the south-west, along the south coast, and on the North Wales coast. For example, in the Mid-Downs and Chichester Districts, private nursing home bed provision was approximately three times greater than NHS geriatric bed provision. ${ }^{31}$ There is not sufficient information on the use of these facilities to determine their precise impact on rates of hospital utilisation. However, HAA data for the South East Thames Region in 1982 show that $5.0 \%$ of surgical discharges in the Region were transferred to another hospital or convalescent unit, the transfer rate for individual Districts ranging from $2.4 \%$ to $10.1 \%$. Transfer rates are likely to be highest among patients with the greatest social needs for care. As a result their hospital use as recorded in HAA will under-represent their total length of stay.
The private acute sector now also plays a significant role in the provision of hospital care. A study based on samples of hospital records in 148 out of the 153 independent hospitals with operating theatres in England and Wales, in 1981, indicated that $13.2 \%$ of all elective surgery (excluding abortions and operations on foreign citizens) was undertaken privately: $9.5 \%$ in the independent sector and $3.8 \%$ in NHS pay beds. ${ }^{31}$ Proportions were much higher for some operations. Thus more than one fifth of all domestic elective total hip replacements, haemorrhoidectomies, hysterectomies, and varicose vein ligation and stripping were undertaken privately in 1981. Since 1981 activity in the independent sector has increased, with a $28 \%$ increase in beds. ${ }^{32}$

Private medical insurance is concentrated among the higher social classes. Over $20 \%$ of men and women in socioeconomic group 1 (professional) hold medical insurance compared with under $2 \%$ in socioeconomic group 6 (unskilled manual). ${ }^{34}$ There is also a marked geographical concentration. The provision of private acute beds ranges from 3 per 1000 population in the Northern Region to 46 per 1000 in North East Thames, and overall $54 \%$ of private acute beds are in the four Thames Regions. ${ }^{35}$ The presence of the private sector and its unequal distribution makes NHS utilisation difficult to interpret but is certain to alter it. It may, for example, have the effect of accentuating the social class gradient of NHS utilisation, particularly in the Thames Regions, through removing some of the affluent from the NHS and introducing a relatively higher utilisation rate by the manual social classes.

\section{Development of utilisation based measures}

Measures of need based on the relative hospital utilisation rates of social groups have so far ignored the effects both of alternative provision and of variations in patient and physician behaviours in translating need into NHS hospital use, despite the importance of these factors on hospitalisation rates at a small area and District level. Attempts have been made to control for the effects of geographical variations in bed provision. However, the methods employed have been fairly crude and do not allow adequately for differences in the availability of hospital beds. As a result the 'deprivation' weights obtained when supply is 'controlled' are not very different from the uncontrolled weights, the hospital utilisation rates of social groups continuing to reflect their access to hospital beds. ${ }^{8}$

Alternative methods have recently been proposed for developing utilisation based measures of need employing spatial interaction models. These are seen as a way of allowing more adequately for the effects of 
supply on the utilisation of NHS hospital beds. Spatial interaction models were developed originally to assist in health service planning. ${ }^{36,37}$ For this purpose they enable planners to predict mathematically how proposed changes in resources and in the supply of facilities in different geographical areas are likely to alter the existing pattern of patient flows. The precise form of each individual model varies depending on the assumptions used. However, they are generally based on the hypothesis that the flow of patients (use) from an area to a particular hospital is a function of:

(1) the relative morbidity of the area population (usually for simplicity of data collection based on the age/sex structure and selected socioeconomic characteristics of the population),

(2) the capacity of the relevant hospital serving the area population; and

(3) the distance of the hospital from the population in the area, allowing for the deterrent effect of increasing distance on use (ie, the fact that use rates tend to decline the further away from the hospital people live).

Whereas the familiar application of spatial interaction models is to predict the pattern of patient flows which would follow the implementation of plans to change the location and scale of facilities, in the current context, the starting point is the observed pattern of utilisation across geographical areas. The objective is to explain this pattern statistically. The variables taken into account in the analysis are 'need' factors (such as population size, age/sex structure, and socioeconomic features) as well as differences in the accessibility of populations in different localities to the supply of hospital beds. In this way, it is argued, it should be possible to assess the proportion of the observed variation in utilisation between areas accounted for by relative accessibility to services and that caused by variations in need.

Spatial interaction models require large data sets, often involving census statistics at a small area level. Defining and quantifying the various elements in the model is complex and involves a series of assumptions. For example, accessibility variables can also be generated in different ways with differing results. The simplest method is to measure the straight line distance of patients from the relevant hospital in an area. However, since the implications of distance vary between urban and rural areas, estimates of travel time are generally preferred but are more laborious to assemble and rely on various assumptions about modes of transport and road conditions. Although these models can take into account all hospitals in the system and the different levels of supply at each hospital, it may be necessary also to take into account the fact that some hospitals, for example teaching hospitals, may draw patients from greater distances than other hospitals. Another problem relates to the choice of socioeconomic indicators as measures of need. As work by the North West Thames Regional Health Authority ${ }^{38}$ has shown, the use of different social classifications can have a significant impact on the 'deprivation weights' assigned to individual Districts, and hence on the calculation of financial targets.

More fundamental than these issues of measurement is the adequacy of spatial interaction models in controlling for the effects of supply. Although affording a more sophisticated approach compared with earlier attempts to control for supply, these models still do not separate out the effects of need on utilisation rates. This is because the relation between each element in the model (ie, the calibration of the model) can only be determined by the existing pattern of utilisation, which is, for the most part, a product of the prevailing distribution of supply. For example, it is only possible to quantify the effect of increasing travel time on utilisation levels by observing the relation of increasing travel times to current patterns of use. There is thus a circularity in the reasoning surrounding the use of spatial interaction models of utilisation. It is simply not possible to specify the relations within the model in order to? identify the proportion of use which is due to "need 2 rather than 'supply' without reference to the existing pattern of use.

\section{Conclusions}

Hospital utilisation data are flawed as an indicator of need for health care, since hospital use is as much a product of the supply of services and professional decisions as any notion of population need. Current utilisation thus provides no indication of the appropriateness of different rates of hospital use among socioeconomic groups or of the distribution of unmet needs. Instead, the amount of hospital care people receive is heavily influenced by the organisation and provision of health services in the area where they live. Hospital admission rates will therefore bear a close relation to the incidence of morbidity only for the fairly small number of conditions that can be diagnosed with reasonable certainty, and for which a consensus exists within the medical profession on the need to treat the illness on an inpatient basis. Broad utilisation based measures of population needs, although derived from increasingly sophisticated modelling procedures, will thus always reflect current patterns of service provision. As a result their use in distributing resources between geographical areas will lead to a situation reminiscent of the pre-RAWP era of the Crossman Formula (1971-75), which based target revenue allocations on 
three elements: the Region's population (given an arbitrary double weight), its beds, and its hospital cases. ${ }^{39}$

One way of improving on the use of mortality data as the need proxy in RAWP after the age and sex structure of the population has been allowed for is to address directly questions of the distribution of morbidity, rather than relying on ever more indirect proxies for health resource need. An issue requiring investigation is the contention that the relation between levels of morbidity and mortality is not a linear 1:1 relation as currently assumed by RAWP. Of particular importance would be to establish the prevalence of functional restrictions and of chronic conditions which place considerable demands on the health services, although only rarely leading to death. The recent Health and Lifestyle Survey with its national sample has shown that individuals' general assessments of their own health correlate well with evidence for the incidence of a wide range of symptoms and the results of more formal physical tests. ${ }^{40}$ This suggests that one approach would be to conduct sample surveys representative of different socioeconomic groups in various Regions of the country. This could be complemented by the inclusion of self reported morbidity questions in the 1991 national census. Another way of improving morbidity data is to mount epidemiological studies of the population prevalence of selected common conditions requiring hospital care for which mortality is unlikely to be an adequate proxy of prevalence. Thirdly, routine systems of data collection might be extended, including the national morbidity survey in general practice and local disease registers.

The search for an alternative to SMRs as a measure of need is often portrayed as a technical problem, the aim being to identify a more adequate measure of morbidity at a population level. However, a fundamental issue which frequently underlies criticisms of SMRs is whether 'need' for hospital resources should be defined solely in terms of morbidity, or whether the definition of need should also include the wider effects of deprivation and geographical variations in the level of primary care provision and of alternative inpatient facilities, since these both directly influence demands on the hospital service, especially at District level. The current approach of employing utilisation data avoids addressing these difficult questions. It merely equates need with that which is measured and, to a greater or lesser extent, reflects current rates of use. An essential prerequisite for the development of measures of need for resource allocation is thus to address directly this central issue of the nature of need and the extent to which different dimensions of need should be compensated for, at least in the short term, through the provision of additional resources in the hospital sector.

\section{References}

${ }^{1}$ Department of Health and Social Security. Sharing resources for health in England: Report of the Resource Allocation Working Party. London: DHSS: 1976.

${ }^{2}$ Buxton MJ, Klein RE. Allocation of health resources: a commentary on the report of the Resource Allocation Working Party. Royal Commission on the NHS. Research Paper No. 3. London: HMSO: 1978.

${ }^{3}$ Woods KJ. Social deprivation and resource allocation in the Thames Regional Health Authorities. In: Health Research Group contemporary perspectives on health and health care. Occasional Paper No. 20, University of London, Queen Mary College, Department of Geography and Earth Science, London, 1982.

${ }^{4}$ Mays N. Measuring need in the National Health Service Resource Allocation Working Party formula: standardised mortality ratios or social deprivation? Public Administration 1987; 65: 45-60.

${ }^{5}$ Department of Health and Social Security. review of the Resource Allocation Working Party formula: Report by the NHS Management Board, London: DHSS: 1986.

${ }^{6}$ McPherson K, Strong PM, Epstein A, Jones L. Regional variations in the use of common surgical procedures: within and between England and Wales, Canada and the United States. Soc Sci Med 1981; 15A: 273-88.

${ }^{7}$ London Health Planning Consortium. Acute hospital services in London: A profile by the London Health Planning Consortium. London: HMSO: 1979.

${ }^{8}$ South East Thames RHA. ACORN: an allowance for social factors in $R A W P$ : consultative paper. Bexhill-on-Sea: SETRHA Statistics and OR Division; 1986.

${ }^{9}$ Department of Health and Social Security. Report of $a$ study of the acute hospital sector. London, 1983.

${ }^{10}$ Morgan M, Paul E, Devlin HB. Length of stay for common surgical procedures: variation among Districts. Br J Surg; 1987; 74: 884-9.

11 Coulter A, McPherson K. The hysterectomy debate. J Soc Affairs 1986; 2(4): 379-96.

12 Black N. Geographical variations in use of surgery for glue ear. J R Soc Med 1985; 78: 641-5.

${ }^{13}$ Sanderson $H$. Regional variation in cataract extraction rates and their relationship with resource supply and need. $J R$ Soc Med 1981; 73: 492-6.

14 Vayda E. Comparison of surgical rates in Canada and in England and Wales. New Eng J Med 1973; 289: 1224-9.

${ }^{15}$ Cooper M. Rationing health care. London; Croom Helm: 1975.

${ }^{16}$ Acheson RM. Variations in hospital referrals. In: G Teeling Smith (ed). Health, education and general practice. London; Office of Health Economics 1986.

${ }^{17}$ Feldstein MS. Economic analysis of health service efficiency. Amsterdam; North Holland Publishing Co. 1967.

${ }^{18}$ Logan RFL, Ashley JSA, Klein RE, Robson DM. Dynamics of medical care: The Liverpool Study into use of hospital resources. Memoir No. 14. London School of Hygiene and Tropical Medicine, 1972.

${ }^{19}$ Wennberg J, Freeman JL, Culp WJ. Are hospital services rationed in New Haven or over-utilised in Boston? Lancet 1987; 23: $1185-9$.

${ }^{20}$ Darby S, Kendall GM, Rae S. The association between frequency of diagnostic radiological examinations and allocation of resources in the National Health Service. Health Trends 1981; 13: 73-5. 
${ }^{21}$ Royal College of General Practitioners Birmingham Research Unit. Practice activity analysis: referrals to specialists. J R Coll Gen Pract 1978; 28: 251-2.

22 Crombie DL, Fleming DM. The background philosophy and potential of practice activity analysis. Birmingham; RCGP Research Unit: 1984.

${ }^{23}$ Wilkin D, Smith A. Variation in GP referrals to consultants. A report prepared for the DHSS. Centre for Primary Care Research, Department of General Practice: University of Manchester, 1986.

${ }^{24}$ Cummins PO, Jarman B, White PM. Do general practitioners have different referral thresholds? Br Med J 1981; 282: 1037-9.

${ }^{25}$ Morrell D, Gage H, Robinson N. Referral to hospital by general practitioners. J R Coll Gen Pract 1970; 19: 331-42.

${ }^{26}$ Dowie R. General practitioners and consultants: a study of outpatient referrals. London; King Edward's Hospital Fund for London 1983.

${ }^{27}$ Bloor M, Venters G. An epidemiological and sociological study of variations in the incidence of operations on the tonsils and adenoids. University of Aberdeen, Institute of Medical Sociology, Occasional Paper No. 2, 1978.

28 Wennberg J. Which rate is right? New Eng J Med 1986: 314: 310-11.

${ }^{29}$ Blaxter M. Equity and consultation rates in general practice. Br Med J 1984; 288: 1963-7.

${ }^{30}$ Hartley RM, Charlton JR, Harris CM, Jarman J. Influence of patient characteristics on test ordering in general practice. Br Med J 1984; 289: 735-8.
${ }^{31}$ Larder D, Day P, Klein R. Institutional care for the elderly: the geographical distribution of the public/private mix in England. Bath: University of Bath, Centre for Policy Analysis. 1986.

32 Williams BT, Nicholl JP, Thomas RJ, Knowelden J. Analysis of the work of independent acute hospitals in England and Wales. Br Med J 1981; 289: 446-8.

${ }^{33}$ Laing W. Private health care. London: Office of Health Economics: 1985.

${ }^{34}$ Office of Population Censuses and Surveys. General household survey, 1983. London: HMSO: 1985.

${ }^{35}$ Rayner G. Private hospital treatment. In: A Harrison, J Gretton (eds) Health care UK, London: Chartered Institute of Public Finance and Accountancy 1986.

${ }^{36}$ Mayhew L. Urban hospital location. London: George Allen and Unwin, 1986.

${ }^{37}$ Taket AR, Mayhew L, Gibberd RW, et al. RAMOS: a model of the spatial allocation of health care resources. In: Health projections in Europe: methods and applications. Copenhagen: World Health Organisation, 1986.

38 North West Thames RHA. Hospital in-patient census, 1981: analysis of the data. London: NWTRHA, Statistics and Health Information Section (no date).

39 West PA. Allocation and equity in the public sector: the Hospital Revenue Allocation Formula. Appl Econ 1973; 5: 153-66.

40 Cox BD, et al. The health and lifestyle survey. London: Health Promotion Trust 1987.

Accepted for publication August 1987 